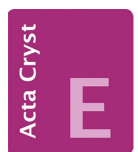

CRYSTALLOGRAPHIC COMMUNICATIONS

ISSN 2056-9890

Received 4 June 2016

Accepted 30 June 2016

Edited by G. Smith, Queensland University of Technology, Australia

Keywords: crystal structure; isoxazolidine; hydrogen bonding.

CCDC reference: 1489217

Supporting information: this article has supporting information at journals.iucr.org/e

\section{Crystal structure of 2-isopropyl-5, $7^{\prime}$-dimethyl- $1^{\prime}, 3^{\prime}, 3 a^{\prime}, 6^{\prime}, 8 a^{\prime}, 8 b^{\prime}$-hexahydrospiro[cyclohexane- $1,6^{\prime}$-furo[3,4-d] imidazo[1,5-b]isoxazol]-8' $\left(7^{\prime} H\right)$-one}

\author{
Heithem Abda, ${ }^{\text {a* }}$ Khaireddine Ezzayani, ${ }^{\text {b }}$ Kaiss Aouadi, ${ }^{a}$ Taha Guerfel, ${ }^{\mathrm{c}}$ Sebastien \\ Vidal $^{\mathrm{d}}$ and Moncef Msaddek $^{\mathrm{a}}$
}

\begin{abstract}
a University of Monastir, Heterocyclic Chemistry Laboratory, Products, Natural and Reactivity, Faculty of Sciences of Monastir, Avenue of the Environment, 5000 Monastir, Tunisia, ${ }^{\mathbf{b}}$ University of Monastir, Laboratory of Physical Chemistry of Materials, Faculty of Sciences of Monastir, Avenue of the Environment, 5019 Monastir, Tunisia, ' Laboratory of

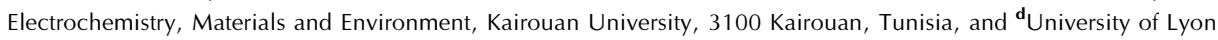
CNRS, Institute of Chemistry and Biochemistry and Molecular Supramolecular, UMR 5246, Laboratoire de Chimie Organique 2-Glycochemistry, Curien Building, 43 Boulevard du 11 Novembre 1918, F-69622 Villeurbanne, France. *Correspondence e-mail: abda_he@hotmail.fr
\end{abstract}

In the title compound, $\mathrm{C}_{17} \mathrm{H}_{28} \mathrm{~N}_{2} \mathrm{O}_{3}$, the isoxazolidine ring adopts an envelope conformation with the $\mathrm{O}$ atom deviating from the mean plane of the other four ring atoms by 0.617 (1) $\AA$. In the crystal, molecules are linked via weak $\mathrm{C}-$ $\mathrm{H}$. O hydrogen bonds, forming chains which extend along the $b$-axis direction.

\section{Chemical context}

The 1,3-dipolar cycloaddition of nitrones to alkenes has been applied to produce substituted isoxazolidines (Gothelf \& Jørgensen, 1998). These compounds can be converted into $\beta$-amino alcohols (Padwa et al., 2002), $\beta$-lactams (Hanselmann et al., 2003) and $\alpha$-amino acids (Aouadi et al., 2006), by reductive cleavage of the $\mathrm{N}-\mathrm{O}$ bond. Consequently, isoxazolidines have been used as key intermediates for the synthesis of various natural products or antifungal, antiinflammatory, anti-mycobacterial, anti-tuberculosis and antiviral agents. The previously mentioned importance of the isoxazolidine substructure led us to investigate the cycloaddition of chiral nitrone [(5(S),6(S),9(R)-6-isopropyl-4,9dimethyl-3-oxo-1,4-diazaspiro[4.5]dec-1-ene-1-oxide] with 2,5-dihydrofuran. The present work reports the synthesis and the X-ray crystallographic study of this substituted isoxazolidine, the title compound, $\mathrm{C}_{17} \mathrm{H}_{28} \mathrm{~N}_{2} \mathrm{O}_{3}$, (I).

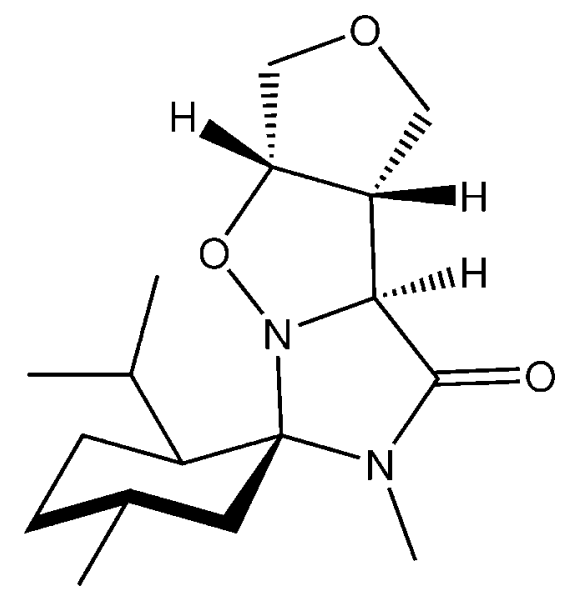




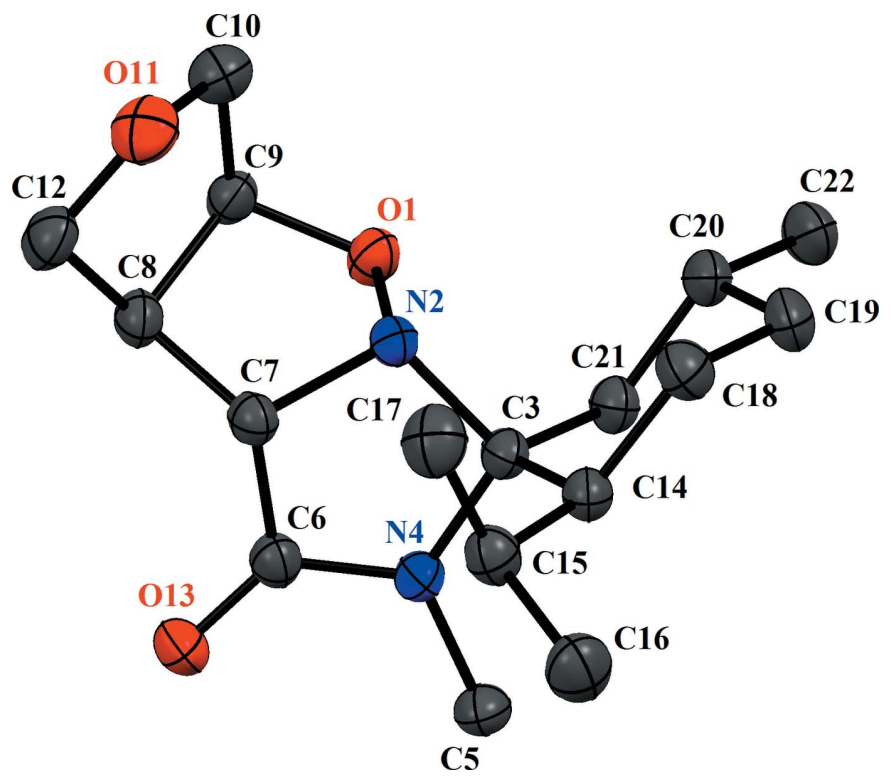

Figure 1

The molecular conformation in the molecules of (I), showing the atom labelling. Displacement ellipsoids are drawn at the $35 \%$ probability level. $\mathrm{H}$ atoms have been omitted for clarity.

\section{Structural commentary}

In the title compound (I), the asymmetric unit comprises a single molecule (Fig. 1). Each molecule has six stereogenic centres (Abda et al., 2014) although the absolute configuration
Table 1

Hydrogen-bond geometry $\left(\AA,^{\circ}\right)$.

\begin{tabular}{lllll}
\hline$D-\mathrm{H} \cdots A$ & $D-\mathrm{H}$ & $\mathrm{H} \cdots A$ & $D \cdots A$ & $D-\mathrm{H} \cdots A$ \\
\hline $\mathrm{C} 5-\mathrm{H} 52 \cdots \mathrm{O} 13^{\mathrm{i}}$ & 0.97 & 2.57 & $3.536(3)$ & 172 \\
\hline
\end{tabular}

Symmetry code: (i) $-x+1, y-\frac{1}{2},-z+\frac{1}{2}$.

for the molecule was not determined definitively in this analysis. The isoxazolidine ring (O1/N2/C7-C9) adopts an envelope conformation with atom O1 displaced by 0.617 (1) $\AA$ from the mean plane through atoms $\mathrm{N} 2 / \mathrm{C} 7-\mathrm{C} 9$. The $\mathrm{N}-\mathrm{O}$ bond lengths of the isoxazolidine rings $\mathrm{O} 1-\mathrm{N} 2=1.482(2) \AA$, close to values reported for related compounds (Loh et al., 2010; Molander et al., 2013).

\section{Supramolecular features}

In the crystal, the molecules are linked via non-classical weak $\mathrm{C} 5-\mathrm{H} 52 \cdots \mathrm{O} 13^{\mathrm{i}}$ hydrogen bonds, forming zigzag chains, which extend along the $b$-axis direction (Table 1 and Fig. 2).

\section{Synthesis and crystallization}

In a Biotage Initiator $10 \mathrm{ml}$ vial, nitrone $[(5(S), 6(S), 9(R)-6$ isopropyl-4,9-dimethyl-3-oxo-1,4-diazaspiro[4.5]dec-1-ene-1oxide] (1 eq.) in anhydrous toluene $(4 \mathrm{ml})$ was introduced.

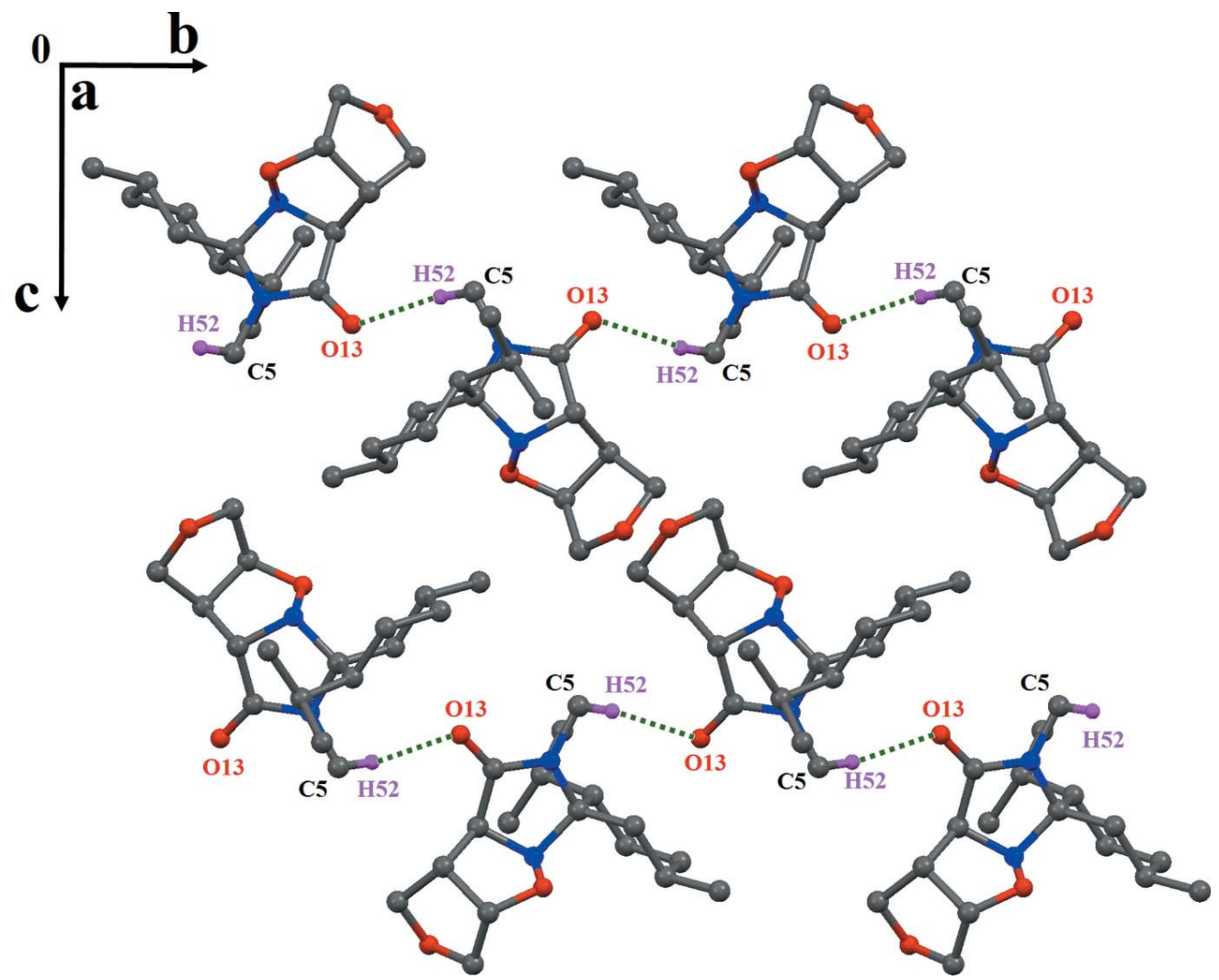

Figure 2

The $\mathrm{C}-\mathrm{H} \cdots \mathrm{O}$ hydrogen-bonded chains extending along the $b$ axis in the crystal structure of (I). Dashed lines indicate hydrogen bonds. Non-associated $\mathrm{H}$ atoms have been omitted. 


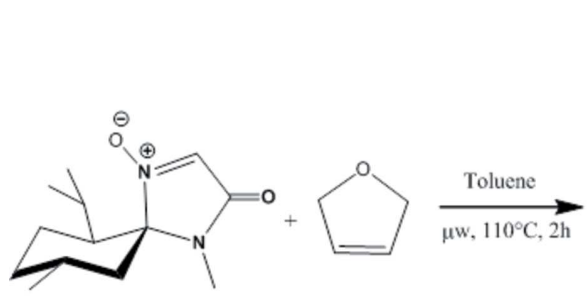

Figure 3

The cycloaddition reaction in the synthesis of (I).

The vial was flushed with argon and 2,5-dihydrofuran (3 eq,) was added. The vial was sealed with a septum cap and was irradiated with microwaves (temperature: $373 \mathrm{~K}$ ) (Fig. 3). TLC monitoring (EtOAc/PE 5/5) showed full conversion after $2 \mathrm{~h}$. After the crude mixture was concentrated and purified by flash column chromatography (silica gel, EtOAc/PE 5/5), the desired isoxazolidine (I) was obtained (m.p. = 410-411 K).

\section{Refinement}

Crystal data, data collection and structure refinement details are summarized in Table 2. The $\mathrm{H}$ atoms were located in a difference map, but these were repositioned geometrically and were initially refined with soft restraints on the bond lengths and angles to regularize their geometry $(\mathrm{C}-\mathrm{H}$ in the range 0.93-0.98 $\AA$ ) and $U_{\text {iso }}(\mathrm{H})$ (in the range 1.2-1.5 times $U_{\text {eq }}$ of the parent atom). These were subsequently refined with riding constraints (Cooper et al., 2010). Although not definitive for this chiral structure, the Flack (1983) absolute structure parameter obtained [0.60 (3) for 1261 Friedel pairs] gave $\mathrm{C} 3(S), \mathrm{C} 7(S), \mathrm{C} 8(S), \mathrm{C} 9(S), \mathrm{C} 14(S), \mathrm{C} 20(R)$ assignments for the six arbitrarily named chiral centres in the molecule. The inverted structure gave a similarly high Flack factor .

\section{Acknowledgements}

The authors are grateful to the Ministry of Higher Education and Scientific Research of Tunisia for financial support.

\section{References}

Abda, H., Aouadi, K., Perrin, L., Msaddek, M., Praly, J.-P. \& Vidal, S. (2014). Eur. J. Org. Chem. pp. 6017-6024.

Agilent (2013). CrysAlis PRO. Agilent Technologies, Yarnton, England.

Altomare, A., Burla, M. C., Camalli, M., Cascarano, G. L., Giacovazzo, C., Guagliardi, A., Moliterni, A. G. G., Polidori, G. \& Spagna, R. (1999). J. Appl. Cryst. 32, 115-119.

Aouadi, K., Vidal, S., Msaddek, M. \& Praly, J.-P. (2006). Synlett, 2006, 3299-3303.

Betteridge, P. W., Carruthers, J. R., Cooper, R. I., Prout, K. \& Watkin, D. J. (2003). J. Appl. Cryst. 36, 1487.

Clark, R. C. \& Reid, J. S. (1995). Acta Cryst. A51, 887-897.

Table 2

Experimental details.

Crystal data

Chemical formula

$M_{\mathrm{r}}$

Crystal system, space group

Temperature (K)

$a, b, c(\AA)$

$V\left(\AA^{3}\right)$

$Z$

Radiation type

$\mu\left(\mathrm{mm}^{-1}\right)$

Crystal size (mm)

Data collection

Diffractometer

Absorption correction

$T_{\min }, T_{\max }$

No. of measured, independent and observed $[I>2.0 \sigma(I)]$ reflections

$R_{\text {int }}$

$(\sin \theta / \lambda)_{\max }\left(\AA^{-1}\right)$

Refinement

$R\left[F^{2}>2 \sigma\left(F^{2}\right)\right], w R\left(F^{2}\right), S$

No. of reflections

No. of parameters

$\mathrm{H}$-atom treatment

$\Delta \rho_{\max }, \Delta \rho_{\min }\left(\mathrm{e} \AA^{-3}\right)$

Absolute structure

Absolute structure parameter

Computer programs: CrysAlis PRO (Agilent, 2013), SIR97 (Altomare et al., 1999), CRYSTALS (Betteridge et al., 2003), CAMERON (Watkin et al., 1996), Larson (1970), Prince (1982) and Watkin (1994).

Cooper, R. I., Thompson, A. L. \& Watkin, D. J. (2010). J. Appl. Cryst. 43, 1100-1107.

Flack, H. D. (1983). Acta Cryst. A39, 876-881.

Görbitz, C. H. (1999). Acta Cryst. B55, 1090-1098.

Gothelf, K. V. \& Jørgensen, K. A. (1998). Chem. Rev. 98, 863-910.

Hanselmann, R., Zhou, J., Ma, P. \& Confalone, P. N. (2003). J. Org. Chem. 68, 8739-8741.

Larson, A. C. (1970). Crystallographic Computing, edited by F. R. Ahmed, pp. 291-294. Copenhagen: Munksgaard.

Loh, B., Vozzolo, L., Mok, B. J., Lee, C. C., Fitzmaurice, R. J., Caddick, S. \& Fassati, A. (2010). Chem. Biol. Drug Des. 75, 461-474.

Molander, G. A., Cavalcanti, L. N. \& García-García, C. (2013). J. Org. Chem. 78, 6427-6439.

Padwa, A. \& Pearson, W. H. (2002). Editors. Synthetic Applications of 1,3-Dipolar Cycloaddition Chemistry Toward Heterocycles and Natural Products, Vol. 59, pp. 1-81. Chichester: Wiley.

Prince, E. (1982). In Mathematical Techniques in Crystallography and Materials Science. New York: Springer-Verlag.

Watkin, D. (1994). Acta Cryst. A50, 411-437.

Watkin, D. J., Prout, C. K. \& Pearce, L. J. (1996). CAMERON. Chemical Crystallography Laboratory, Oxford, England. 


\section{supporting information}

Acta Cryst. (2016). E72, 1150-1152［https://doi.org/10.1107/S2056989016010641]

Crystal structure of 2-isopropyl-5, $7^{\prime}$-dimethyl-1', $3^{\prime}, 3 \mathrm{a}^{\prime}, 6^{\prime}, 8 \mathrm{a}^{\prime}, 8 \mathrm{~b}^{\prime}$-hexahydrospiro[cyclohexane-1, $6^{\prime}$-furo[3,4-d]imidazo[1,5-b] isoxazol]-8' $\left(7^{\prime} H\right)$-one

Heithem Abda, Khaireddine Ezzayani, Kaiss Aouadi, Taha Guerfel, Sebastien Vidal and Moncef

Msaddek

Computing details

Data collection: CrysAlis PRO (Agilent, 2013); cell refinement: CrysAlis PRO (Agilent, 2013); data reduction: CrysAlis PRO (Agilent, 2013); program(s) used to solve structure: SIR97 (Altomare et al., 1999); program(s) used to refine structure: CRYSTALS (Betteridge et al., 2003); molecular graphics: CAMERON (Watkin et al., 1996); software used to prepare material for publication: CRYSTALS (Betteridge et al., 2003).

(I)

Crystal data

$\mathrm{C}_{17} \mathrm{H}_{28} \mathrm{~N}_{2} \mathrm{O}_{3}$

$M_{r}=308.42$

Orthorhombic, $P 2_{1} 2_{1} 2_{1}$

Hall symbol: P 2ac 2ab

$a=7.7474(6) \AA$

$b=11.1404(8) \AA$

$c=19.208(2) \AA$

$V=1657.8(2) \AA^{3}$

$Z=4$

$F(000)=672$

$D_{\mathrm{x}}=1.236 \mathrm{Mg} \mathrm{m}^{-3}$

$\mathrm{Cu} K \alpha$ radiation, $\lambda=1.5418 \AA$

Cell parameters from 5548 reflections

$\theta=4.5-66.7^{\circ}$

$\mu=0.68 \mathrm{~mm}^{-1}$

$T=150 \mathrm{~K}$

Block, colorless

$0.49 \times 0.43 \times 0.25 \mathrm{~mm}$

\section{Data collection}

Oxford Diffraction Xcalibur (Atlas, Gemini Ultra)

diffractometer

Radiation source: Enhance Ultra $(\mathrm{Cu}) \mathrm{X}$-ray source

Mirror monochromator

Detector resolution: 10.4678 pixels $\mathrm{mm}^{-1}$

$\omega$ scans

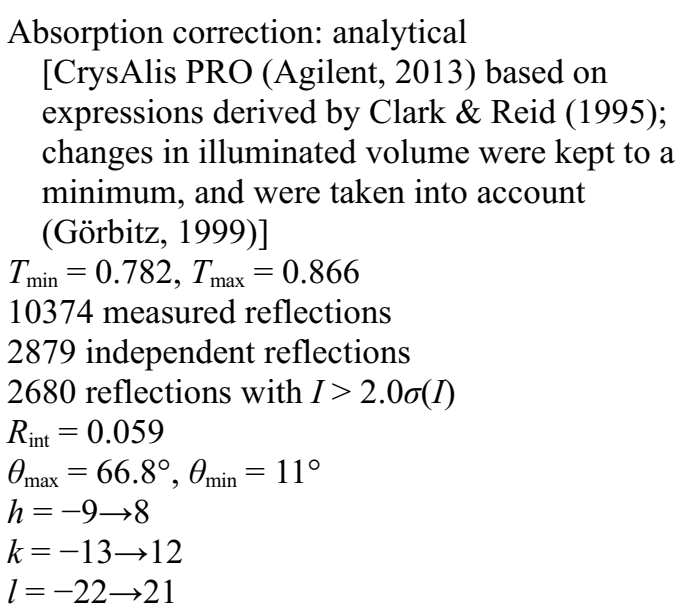




\section{Refinement}

Refinement on $F^{2}$

Least-squares matrix: full

$R\left[F^{2}>2 \sigma\left(F^{2}\right)\right]=0.042$

$w R\left(F^{2}\right)=0.096$

$S=1.03$

2866 reflections

201 parameters

0 restraints

Primary atom site location: structure-invariant direct methods

Hydrogen site location: difference Fourier map $\mathrm{H}$-atom parameters constrained
Method, part 1, Chebychev polynomial, (Watkin, 1994: Prince, 1982) [weight] $=$ $\left.1.0 /\left[\mathrm{A}_{0} * \mathrm{~T}_{0}(\mathrm{x})+\mathrm{A}_{1} * \mathrm{~T}_{1}(\mathrm{x}) \cdots+\mathrm{A}_{\mathrm{n}-1}\right] * \mathrm{~T}_{\mathrm{n}-1}(\mathrm{x})\right]$ where $\mathrm{A}_{\mathrm{i}}$ are the Chebychev coefficients listed below and $\mathrm{x}=F / F \max$ Method $=$ Robust Weighting (Prince, 1982) W $=[$ weight $] *$ [1-(delta $F / 6 *$ sigma $\left.F)^{2}\right]^{2} \mathrm{~A}_{\mathrm{i}}$ are: $0.124 \mathrm{E}+04$ $0.195 \mathrm{E}+040.105 \mathrm{E}+04304$.

$(\Delta / \sigma)_{\max }=0.0002$

$\Delta \rho_{\max }=0.16 \mathrm{e} \AA^{-3}$

$\Delta \rho_{\min }=-0.17$ e $\AA^{-3}$

Extinction correction: Larson (1970), Equation 22

Extinction coefficient: 74 (4)

Absolute structure: Flack (1983), 1261 Friedel pairs

Absolute structure parameter: $0.6(3)$

\section{Special details}

Experimental. The crystal was placed in the cold stream of an Oxford Cryosystems open-flow nitrogen cryostat with a nominal stability of $0.1 \mathrm{~K}$.

Refinement. The analytical numeric absorption correction using a multi-faceted crystal model is based on expressions derived by Clark \& Reid (1995). The relatively large ratio of minimum to maximum corrections applied in the multi-scan process (1:nnn) reflect changes in the illuminated volume of the crystal. Changes in illuminated volume were kept to a minimum, and were taken into account (Görbitz, 1999).

Fractional atomic coordinates and isotropic or equivalent isotropic displacement parameters $\left(\hat{A}^{2}\right)$

\begin{tabular}{lllll}
\hline & $x$ & $y$ & $z$ & $U_{\text {iso }} / U_{\text {eq }}$ \\
\hline O1 & $0.3924(2)$ & $0.46528(13)$ & $0.43226(8)$ & 0.0295 \\
N2 & $0.2276(2)$ & $0.48439(15)$ & $0.39468(9)$ & 0.0248 \\
C3 & $0.2216(3)$ & $0.39172(18)$ & $0.33856(11)$ & 0.0253 \\
N4 & $0.3084(3)$ & $0.44977(16)$ & $0.27929(9)$ & 0.0257 \\
C5 & $0.3388(3)$ & $0.3911(2)$ & $0.21315(11)$ & 0.0309 \\
H51 & 0.3934 & 0.4467 & 0.1812 & $0.0471^{*}$ \\
H53 & 0.2321 & 0.3682 & 0.1919 & $0.0466^{*}$ \\
H52 & 0.4129 & 0.3213 & 0.2193 & $0.0470^{*}$ \\
C6 & $0.3369(3)$ & $0.56800(19)$ & $0.28913(11)$ & 0.0269 \\
C7 & $0.2626(3)$ & $0.60075(18)$ & $0.35923(11)$ & 0.0257 \\
C8 & $0.3798(3)$ & $0.67280(19)$ & $0.40726(12)$ & 0.0279 \\
C9 & $0.4274(3)$ & $0.5792(2)$ & $0.46276(11)$ & 0.0271 \\
C10 & $0.3137(4)$ & $0.6110(2)$ & $0.52465(12)$ & 0.0372 \\
O11 & $0.1969(2)$ & $0.70248(16)$ & $0.50158(9)$ & 0.0397 \\
C12 & $0.2873(3)$ & $0.7679(2)$ & $0.44941(13)$ & 0.0352 \\
H122 & 0.2094 & 0.8163 & 0.4202 & $0.0417^{*}$ \\
H121 & 0.3689 & 0.8236 & 0.4714 & $0.0416^{*}$ \\
H102 & 0.2495 & 0.5396 & 0.5410 & $0.0447^{*}$ \\
H101 & 0.3821 & 0.6433 & 0.5635 & $0.0448^{*}$ \\
H91 & 0.5499 & 0.5839 & 0.4753 & $0.0337^{*}$ \\
H81 & 0.4758 & 0.7027 & 0.3812 & $0.0341^{*}$
\end{tabular}




$\begin{array}{lllll}\text { H71 } & 0.1551 & 0.6415 & 0.3518 & 0.0307^{*} \\ \text { O13 } & 0.4052(2) & 0.63710(15) & 0.24780(8) & 0.0357 \\ \text { C14 } & 0.0307(3) & 0.3616(2) & 0.32135(11) & 0.0274 \\ \text { C15 } & -0.0806(3) & 0.4686(2) & 0.29604(12) & 0.0310 \\ \text { C16 } & -0.2129(4) & 0.4250(3) & 0.24307(14) & 0.0435 \\ \text { H162 } & -0.2845 & 0.4937 & 0.2288 & 0.0654^{*} \\ \text { H163 } & -0.1594 & 0.3903 & 0.2017 & 0.0653^{*} \\ \text { H161 } & -0.2850 & 0.3629 & 0.2652 & 0.0658^{*} \\ \text { C17 } & -0.1731(4) & 0.5369(2) & 0.35403(14) & 0.0406 \\ \text { H171 } & -0.2186 & 0.6122 & 0.3336 & 0.0606^{*} \\ \text { H173 } & -0.2672 & 0.4908 & 0.3721 & 0.0604^{*} \\ \text { H172 } & -0.0979 & 0.5542 & 0.3931 & 0.0602^{*} \\ \text { H151 } & -0.0031 & 0.5263 & 0.2711 & 0.0372^{*} \\ \text { C18 } & -0.0536(3) & 0.2924(2) & 0.38122(13) & 0.0346 \\ \text { C19 } & 0.0460(4) & 0.1795(2) & 0.40142(14) & 0.0379 \\ \text { C20 } & 0.2339(3) & 0.2100(2) & 0.41916(12) & 0.0327 \\ \text { C21 } & 0.3154(3) & 0.27553(19) & 0.35817(12) & 0.0288 \\ \text { H211 } & 0.3096 & 0.2212 & 0.3171 & 0.0339^{*} \\ \text { H212 } & 0.4342 & 0.2949 & 0.3684 & 0.0350^{*} \\ \text { C22 } & 0.3387(4) & 0.0976(2) & 0.43654(13) & 0.0429 \\ \text { H222 } & 0.4583 & 0.1173 & 0.4469 & 0.0645^{*} \\ \text { H221 } & 0.3358 & 0.0416 & 0.3963 & 0.0629^{*} \\ \text { H223 } & 0.2912 & 0.0549 & 0.4787 & 0.0629^{*} \\ \text { H201 } & 0.2358 & 0.2644 & 0.4599 & 0.0385^{*} \\ \text { H192 } & 0.0469 & 0.1237 & 0.3607 & 0.0463^{*} \\ \text { H191 } & -0.0113 & 0.1390 & 0.4418 & 0.0451^{*} \\ \text { H182 } & -0.1724 & 0.2721 & 0.3658 & 0.0423^{*} \\ \text { H181 } & -0.0606 & 0.3469 & 0.4217 & 0.0410^{*} \\ \text { H141 } & 0.0390 & 0.3055 & 0.2810 & 0.0325^{*}\end{array}$

Atomic displacement parameters $\left(\AA^{2}\right)$

\begin{tabular}{lllllll}
\hline & $U^{11}$ & $U^{22}$ & $U^{33}$ & $U^{12}$ & $U^{13}$ & $U^{23}$ \\
\hline O1 & $0.0344(8)$ & $0.0232(7)$ & $0.0308(8)$ & $0.0026(7)$ & $-0.0085(7)$ & $-0.0014(6)$ \\
N2 & $0.0304(10)$ & $0.0202(9)$ & $0.0239(9)$ & $0.0002(8)$ & $-0.0028(8)$ & $0.0005(7)$ \\
C3 & $0.0324(12)$ & $0.0197(10)$ & $0.0237(10)$ & $0.0008(9)$ & $0.0029(9)$ & $-0.0011(9)$ \\
N4 & $0.0317(9)$ & $0.0218(9)$ & $0.0236(9)$ & $-0.0001(8)$ & $0.0035(8)$ & $-0.0015(7)$ \\
C5 & $0.0377(12)$ & $0.0307(11)$ & $0.0242(11)$ & $-0.0039(10)$ & $0.0039(9)$ & $-0.0034(9)$ \\
C6 & $0.0290(11)$ & $0.0233(10)$ & $0.0284(11)$ & $-0.0006(9)$ & $-0.0018(9)$ & $0.0016(9)$ \\
C7 & $0.0302(11)$ & $0.0206(11)$ & $0.0262(10)$ & $0.0023(10)$ & $0.0024(9)$ & $0.0014(8)$ \\
C8 & $0.0324(12)$ & $0.0204(10)$ & $0.0307(12)$ & $-0.0016(9)$ & $0.0028(10)$ & $-0.0014(9)$ \\
C9 & $0.0313(11)$ & $0.0233(10)$ & $0.0267(10)$ & $0.0000(9)$ & $-0.0042(9)$ & $-0.0040(9)$ \\
C10 & $0.0453(14)$ & $0.0361(13)$ & $0.0302(12)$ & $-0.0014(12)$ & $-0.0002(11)$ & $-0.0035(10)$ \\
O11 & $0.0392(10)$ & $0.0387(9)$ & $0.0413(9)$ & $0.0061(8)$ & $0.0076(8)$ & $-0.0078(8)$ \\
C12 & $0.0421(15)$ & $0.0257(11)$ & $0.0377(13)$ & $0.0022(11)$ & $-0.0016(11)$ & $-0.0076(10)$ \\
O13 & $0.0489(10)$ & $0.0280(8)$ & $0.0303(8)$ & $-0.0062(8)$ & $0.0057(8)$ & $0.0044(6)$ \\
C14 & $0.0329(11)$ & $0.0228(11)$ & $0.0265(11)$ & $-0.0031(9)$ & $0.0021(9)$ & $-0.0005(9)$ \\
C15 & $0.0295(11)$ & $0.0298(12)$ & $0.0335(12)$ & $-0.0022(10)$ & $-0.0007(10)$ & $0.0024(9)$
\end{tabular}




\begin{tabular}{lllllll}
\hline & & & & & \\
C16 & $0.0430(14)$ & $0.0423(14)$ & $0.0453(14)$ & $-0.0056(13)$ & $-0.0109(13)$ & $0.0027(12)$ \\
C17 & $0.0370(13)$ & $0.0368(13)$ & $0.0481(15)$ & $0.0038(12)$ & $0.0041(12)$ & $-0.0022(11)$ \\
C18 & $0.0371(13)$ & $0.0322(13)$ & $0.0344(12)$ & $-0.0047(11)$ & $0.0039(11)$ & $0.0025(10)$ \\
C19 & $0.0533(16)$ & $0.0238(12)$ & $0.0365(13)$ & $-0.0085(11)$ & $0.0037(12)$ & $0.0039(10)$ \\
C20 & $0.0495(15)$ & $0.0209(11)$ & $0.0278(11)$ & $-0.0015(11)$ & $-0.0011(11)$ & $-0.0001(9)$ \\
C21 & $0.0360(12)$ & $0.0211(11)$ & $0.0292(11)$ & $0.0013(10)$ & $0.0009(10)$ & $-0.0008(9)$ \\
C22 & $0.0680(19)$ & $0.0239(12)$ & $0.0369(13)$ & $0.0025(12)$ & $-0.0068(13)$ & $0.0040(10)$ \\
\hline
\end{tabular}

Geometric parameters $\left(\AA,{ }^{\circ}\right)$

\begin{tabular}{|c|c|c|c|}
\hline $\mathrm{O} 1-\mathrm{N} 2$ & $1.482(2)$ & $\mathrm{C} 14-\mathrm{C} 15$ & $1.550(3)$ \\
\hline $\mathrm{O} 1-\mathrm{C} 9$ & $1.424(3)$ & $\mathrm{C} 14-\mathrm{C} 18$ & $1.531(3)$ \\
\hline $\mathrm{N} 2-\mathrm{C} 3$ & $1.493(3)$ & $\mathrm{C} 14-\mathrm{H} 141$ & 0.997 \\
\hline $\mathrm{N} 2-\mathrm{C} 7$ & $1.489(3)$ & $\mathrm{C} 15-\mathrm{C} 16$ & $1.524(3)$ \\
\hline $\mathrm{C} 3-\mathrm{N} 4$ & $1.472(3)$ & $\mathrm{C} 15-\mathrm{C} 17$ & $1.527(3)$ \\
\hline $\mathrm{C} 3-\mathrm{C} 14$ & $1.552(3)$ & $\mathrm{C} 15-\mathrm{H} 151$ & 1.001 \\
\hline $\mathrm{C} 3-\mathrm{C} 21$ & $1.531(3)$ & $\mathrm{C} 16-\mathrm{H} 162$ & 0.983 \\
\hline $\mathrm{N} 4-\mathrm{C} 5$ & $1.448(3)$ & $\mathrm{C} 16-\mathrm{H} 163$ & 0.977 \\
\hline $\mathrm{N} 4-\mathrm{C} 6$ & $1.349(3)$ & $\mathrm{C} 16-\mathrm{H} 161$ & 0.986 \\
\hline C5-H51 & 0.969 & $\mathrm{C} 17-\mathrm{H} 171$ & 0.991 \\
\hline $\mathrm{C} 5-\mathrm{H} 53$ & 0.956 & $\mathrm{C} 17-\mathrm{H} 173$ & 0.957 \\
\hline $\mathrm{C} 5-\mathrm{H} 52$ & 0.974 & $\mathrm{C} 17-\mathrm{H} 172$ & 0.970 \\
\hline $\mathrm{C} 6-\mathrm{C} 7$ & $1.509(3)$ & $\mathrm{C} 18-\mathrm{C} 19$ & $1.526(4)$ \\
\hline $\mathrm{C} 6-\mathrm{O} 13$ & $1.226(3)$ & $\mathrm{C} 18-\mathrm{H} 182$ & 0.992 \\
\hline $\mathrm{C} 7-\mathrm{C} 8$ & $1.523(3)$ & $\mathrm{C} 18-\mathrm{H} 181$ & 0.988 \\
\hline $\mathrm{C} 7-\mathrm{H} 71$ & 0.959 & $\mathrm{C} 19-\mathrm{C} 20$ & $1.533(4)$ \\
\hline $\mathrm{C} 8-\mathrm{C} 9$ & $1.536(3)$ & $\mathrm{C} 19-\mathrm{H} 192$ & 0.999 \\
\hline $\mathrm{C} 8-\mathrm{C} 12$ & $1.513(3)$ & C19-H191 & 1.001 \\
\hline $\mathrm{C} 8-\mathrm{H} 81$ & 0.957 & $\mathrm{C} 20-\mathrm{C} 21$ & $1.518(3)$ \\
\hline $\mathrm{C} 9-\mathrm{C} 10$ & $1.521(3)$ & $\mathrm{C} 20-\mathrm{C} 22$ & $1.529(3)$ \\
\hline C9-H91 & 0.980 & $\mathrm{C} 20-\mathrm{H} 201$ & 0.990 \\
\hline $\mathrm{C} 10-\mathrm{O} 11$ & $1.433(3)$ & $\mathrm{C} 21-\mathrm{H} 211$ & 0.996 \\
\hline $\mathrm{C} 10-\mathrm{H} 102$ & 0.989 & $\mathrm{C} 21-\mathrm{H} 212$ & 0.966 \\
\hline $\mathrm{C} 10-\mathrm{H} 101$ & 0.983 & $\mathrm{C} 22-\mathrm{H} 222$ & 0.972 \\
\hline $\mathrm{O} 11-\mathrm{C} 12$ & $1.423(3)$ & $\mathrm{C} 22-\mathrm{H} 221$ & 0.995 \\
\hline $\mathrm{C} 12-\mathrm{H} 122$ & 0.985 & $\mathrm{C} 22-\mathrm{H} 223$ & 1.009 \\
\hline $\mathrm{C} 12-\mathrm{H} 121$ & 0.981 & & \\
\hline $\mathrm{N} 2-\mathrm{O} 1-\mathrm{C} 9$ & $103.68(14)$ & $\mathrm{C} 3-\mathrm{C} 14-\mathrm{C} 18$ & $110.82(19)$ \\
\hline $\mathrm{O} 1-\mathrm{N} 2-\mathrm{C} 3$ & $106.18(15)$ & $\mathrm{C} 15-\mathrm{C} 14-\mathrm{C} 18$ & $112.69(19)$ \\
\hline $\mathrm{O} 1-\mathrm{N} 2-\mathrm{C} 7$ & $100.99(15)$ & $\mathrm{C} 3-\mathrm{C} 14-\mathrm{H} 141$ & 103.9 \\
\hline $\mathrm{C} 3-\mathrm{N} 2-\mathrm{C} 7$ & $106.11(15)$ & $\mathrm{C} 15-\mathrm{C} 14-\mathrm{H} 141$ & 105.9 \\
\hline $\mathrm{N} 2-\mathrm{C} 3-\mathrm{N} 4$ & $103.92(16)$ & $\mathrm{C} 18-\mathrm{C} 14-\mathrm{H} 141$ & 107.2 \\
\hline $\mathrm{N} 2-\mathrm{C} 3-\mathrm{C} 14$ & $109.41(17)$ & $\mathrm{C} 14-\mathrm{C} 15-\mathrm{C} 16$ & $109.79(19)$ \\
\hline $\mathrm{N} 4-\mathrm{C} 3-\mathrm{C} 14$ & $111.43(18)$ & $\mathrm{C} 14-\mathrm{C} 15-\mathrm{C} 17$ & $114.5(2)$ \\
\hline $\mathrm{N} 2-\mathrm{C} 3-\mathrm{C} 21$ & $113.10(17)$ & $\mathrm{C} 16-\mathrm{C} 15-\mathrm{C} 17$ & $109.3(2)$ \\
\hline $\mathrm{N} 4-\mathrm{C} 3-\mathrm{C} 21$ & $110.18(18)$ & $\mathrm{C} 14-\mathrm{C} 15-\mathrm{H} 151$ & 108.1 \\
\hline $\mathrm{C} 14-\mathrm{C} 3-\mathrm{C} 21$ & $108.77(18)$ & $\mathrm{C} 16-\mathrm{C} 15-\mathrm{H} 151$ & 106.8 \\
\hline
\end{tabular}




\begin{tabular}{|c|c|c|c|}
\hline $\mathrm{C} 3-\mathrm{N} 4-\mathrm{C} 5$ & $123.70(17)$ & $\mathrm{C} 17-\mathrm{C} 15-\mathrm{H} 151$ & 108.1 \\
\hline $\mathrm{C} 3-\mathrm{N} 4-\mathrm{C} 6$ & $113.29(17)$ & $\mathrm{C} 15-\mathrm{C} 16-\mathrm{H} 162$ & 108.5 \\
\hline $\mathrm{C} 5-\mathrm{N} 4-\mathrm{C} 6$ & $122.47(19)$ & $\mathrm{C} 15-\mathrm{C} 16-\mathrm{H} 163$ & 112.6 \\
\hline $\mathrm{N} 4-\mathrm{C} 5-\mathrm{H} 51$ & 109.8 & $\mathrm{H} 162-\mathrm{C} 16-\mathrm{H} 163$ & 108.7 \\
\hline $\mathrm{N} 4-\mathrm{C} 5-\mathrm{H} 53$ & 110.8 & $\mathrm{C} 15-\mathrm{C} 16-\mathrm{H} 161$ & 108.4 \\
\hline $\mathrm{H} 51-\mathrm{C} 5-\mathrm{H} 53$ & 106.1 & $\mathrm{H} 162-\mathrm{C} 16-\mathrm{H} 161$ & 110.3 \\
\hline $\mathrm{N} 4-\mathrm{C} 5-\mathrm{H} 52$ & 110.4 & $\mathrm{H} 163-\mathrm{C} 16-\mathrm{H} 161$ & 108.3 \\
\hline $\mathrm{H} 51-\mathrm{C} 5-\mathrm{H} 52$ & 109.2 & $\mathrm{C} 15-\mathrm{C} 17-\mathrm{H} 171$ & 107.4 \\
\hline $\mathrm{H} 53-\mathrm{C} 5-\mathrm{H} 52$ & 110.4 & $\mathrm{C} 15-\mathrm{C} 17-\mathrm{H} 173$ & 110.8 \\
\hline $\mathrm{N} 4-\mathrm{C} 6-\mathrm{C} 7$ & $107.39(18)$ & $\mathrm{H} 171-\mathrm{C} 17-\mathrm{H} 173$ & 109.1 \\
\hline $\mathrm{N} 4-\mathrm{C} 6-\mathrm{O} 13$ & $126.4(2)$ & $\mathrm{C} 15-\mathrm{C} 17-\mathrm{H} 172$ & 112.4 \\
\hline $\mathrm{C} 7-\mathrm{C} 6-\mathrm{O} 13$ & $126.2(2)$ & $\mathrm{H} 171-\mathrm{C} 17-\mathrm{H} 172$ & 110.5 \\
\hline $\mathrm{C} 6-\mathrm{C} 7-\mathrm{N} 2$ & $105.48(16)$ & $\mathrm{H} 173-\mathrm{C} 17-\mathrm{H} 172$ & 106.5 \\
\hline $\mathrm{C} 6-\mathrm{C} 7-\mathrm{C} 8$ & $116.15(19)$ & $\mathrm{C} 14-\mathrm{C} 18-\mathrm{C} 19$ & $113.0(2)$ \\
\hline $\mathrm{N} 2-\mathrm{C} 7-\mathrm{C} 8$ & $106.89(17)$ & $\mathrm{C} 14-\mathrm{C} 18-\mathrm{H} 182$ & 106.7 \\
\hline $\mathrm{C} 6-\mathrm{C} 7-\mathrm{H} 71$ & 108.3 & $\mathrm{C} 19-\mathrm{C} 18-\mathrm{H} 182$ & 110.9 \\
\hline $\mathrm{N} 2-\mathrm{C} 7-\mathrm{H} 71$ & 108.7 & $\mathrm{C} 14-\mathrm{C} 18-\mathrm{H} 181$ & 107.8 \\
\hline $\mathrm{C} 8-\mathrm{C} 7-\mathrm{H} 71$ & 111.0 & $\mathrm{C} 19-\mathrm{C} 18-\mathrm{H} 181$ & 109.5 \\
\hline $\mathrm{C} 7-\mathrm{C} 8-\mathrm{C} 9$ & $101.88(16)$ & $\mathrm{H} 182-\mathrm{C} 18-\mathrm{H} 181$ & 108.9 \\
\hline $\mathrm{C} 7-\mathrm{C} 8-\mathrm{C} 12$ & $114.2(2)$ & $\mathrm{C} 18-\mathrm{C} 19-\mathrm{C} 20$ & $110.74(19)$ \\
\hline $\mathrm{C} 9-\mathrm{C} 8-\mathrm{C} 12$ & $102.57(18)$ & $\mathrm{C} 18-\mathrm{C} 19-\mathrm{H} 192$ & 108.4 \\
\hline $\mathrm{C} 7-\mathrm{C} 8-\mathrm{H} 81$ & 109.3 & $\mathrm{C} 20-\mathrm{C} 19-\mathrm{H} 192$ & 107.8 \\
\hline $\mathrm{C} 9-\mathrm{C} 8-\mathrm{H} 81$ & 114.4 & $\mathrm{C} 18-\mathrm{C} 19-\mathrm{H} 191$ & 110.1 \\
\hline $\mathrm{C} 12-\mathrm{C} 8-\mathrm{H} 81$ & 113.9 & $\mathrm{C} 20-\mathrm{C} 19-\mathrm{H} 191$ & 110.4 \\
\hline $\mathrm{C} 8-\mathrm{C} 9-\mathrm{O} 1$ & $105.88(16)$ & $\mathrm{H} 192-\mathrm{C} 19-\mathrm{H} 191$ & 109.2 \\
\hline $\mathrm{C} 8-\mathrm{C} 9-\mathrm{C} 10$ & $104.18(18)$ & $\mathrm{C} 19-\mathrm{C} 20-\mathrm{C} 21$ & $109.3(2)$ \\
\hline $\mathrm{O} 1-\mathrm{C} 9-\mathrm{C} 10$ & $114.75(19)$ & $\mathrm{C} 19-\mathrm{C} 20-\mathrm{C} 22$ & $111.8(2)$ \\
\hline $\mathrm{C} 8-\mathrm{C} 9-\mathrm{H} 91$ & 111.5 & $\mathrm{C} 21-\mathrm{C} 20-\mathrm{C} 22$ & $110.0(2)$ \\
\hline $\mathrm{O} 1-\mathrm{C} 9-\mathrm{H} 91$ & 109.5 & $\mathrm{C} 19-\mathrm{C} 20-\mathrm{H} 201$ & 109.0 \\
\hline $\mathrm{C} 10-\mathrm{C} 9-\mathrm{H} 91$ & 110.8 & $\mathrm{C} 21-\mathrm{C} 20-\mathrm{H} 201$ & 108.1 \\
\hline $\mathrm{C} 9-\mathrm{C} 10-\mathrm{O} 11$ & $106.84(18)$ & $\mathrm{C} 22-\mathrm{C} 20-\mathrm{H} 201$ & 108.7 \\
\hline $\mathrm{C} 9-\mathrm{C} 10-\mathrm{H} 102$ & 110.7 & $\mathrm{C} 3-\mathrm{C} 21-\mathrm{C} 20$ & $113.52(19)$ \\
\hline $\mathrm{O} 11-\mathrm{C} 10-\mathrm{H} 102$ & 110.6 & $\mathrm{C} 3-\mathrm{C} 21-\mathrm{H} 211$ & 107.3 \\
\hline $\mathrm{C} 9-\mathrm{C} 10-\mathrm{H} 101$ & 111.5 & $\mathrm{C} 20-\mathrm{C} 21-\mathrm{H} 211$ & 107.5 \\
\hline $\mathrm{O} 11-\mathrm{C} 10-\mathrm{H} 101$ & 108.3 & $\mathrm{C} 3-\mathrm{C} 21-\mathrm{H} 212$ & 108.2 \\
\hline $\mathrm{H} 102-\mathrm{C} 10-\mathrm{H} 101$ & 108.9 & $\mathrm{C} 20-\mathrm{C} 21-\mathrm{H} 212$ & 110.3 \\
\hline $\mathrm{C} 10-\mathrm{O} 11-\mathrm{C} 12$ & $105.74(19)$ & $\mathrm{H} 211-\mathrm{C} 21-\mathrm{H} 212$ & 109.9 \\
\hline $\mathrm{C} 8-\mathrm{C} 12-\mathrm{O} 11$ & $104.57(19)$ & $\mathrm{C} 20-\mathrm{C} 22-\mathrm{H} 222$ & 111.5 \\
\hline $\mathrm{C} 8-\mathrm{C} 12-\mathrm{H} 122$ & 111.7 & $\mathrm{C} 20-\mathrm{C} 22-\mathrm{H} 221$ & 109.4 \\
\hline $\mathrm{O} 11-\mathrm{C} 12-\mathrm{H} 122$ & 112.4 & $\mathrm{H} 222-\mathrm{C} 22-\mathrm{H} 221$ & 108.8 \\
\hline $\mathrm{C} 8-\mathrm{C} 12-\mathrm{H} 121$ & 111.6 & $\mathrm{C} 20-\mathrm{C} 22-\mathrm{H} 223$ & 111.6 \\
\hline $\mathrm{O} 11-\mathrm{C} 12-\mathrm{H} 121$ & 109.7 & $\mathrm{H} 222-\mathrm{C} 22-\mathrm{H} 223$ & 106.8 \\
\hline $\mathrm{H} 122-\mathrm{C} 12-\mathrm{H} 121$ & 107.0 & $\mathrm{H} 221-\mathrm{C} 22-\mathrm{H} 223$ & 108.7 \\
\hline $\mathrm{C} 3-\mathrm{C} 14-\mathrm{C} 15$ & $115.54(18)$ & & \\
\hline $\mathrm{C} 9-\mathrm{O} 1-\mathrm{N} 2-\mathrm{C} 3$ & $156.12(16)$ & $\mathrm{C} 21-\mathrm{C} 3-\mathrm{C} 14-\mathrm{C} 18$ & $53.8(2)$ \\
\hline $\mathrm{C} 9-\mathrm{O} 1-\mathrm{N} 2-\mathrm{C} 7$ & $45.58(18)$ & $\mathrm{N} 2-\mathrm{C} 3-\mathrm{C} 21-\mathrm{C} 20$ & $64.1(2)$ \\
\hline $\mathrm{N} 2-\mathrm{O} 1-\mathrm{C} 9-\mathrm{C} 8$ & $-40.8(2)$ & $\mathrm{N} 4-\mathrm{C} 3-\mathrm{C} 21-\mathrm{C} 20$ & $179.91(18)$ \\
\hline
\end{tabular}




$73.5(2)$
$-30.4(2)$
$41.4(2)$
$-88.72(18)$
$152.15(15)$
$30.7(2)$
$18.2(2)$
$-100.94(18)$
$137.64(18)$
$91.23(18)$
$-32.94(19)$
$-19.4(2)$
$-143.56(17)$
$177.7(2)$
$-64.6(3)$
$56.2(3)$
$-10.6(3)$
$107.2(2)$
$-132.0(2)$
$-179.3(2)$
$-1.6(3)$
$-7.4(4)$
$170.3(2)$
$59.5(2)$
$-70.2(2)$
$-54.8(2)$
$175.48(17)$
$-176.47(18)$

$73.5(2)$

$\mathrm{C} 12-\mathrm{O} 11-\mathrm{C} 10-\mathrm{C} 9$

$\mathrm{C} 10-\mathrm{O} 11-\mathrm{C} 12-\mathrm{C} 8$

$\mathrm{O} 1-\mathrm{N} 2-\mathrm{C} 3-\mathrm{N} 4$

$\mathrm{O} 1-\mathrm{N} 2-\mathrm{C} 3-\mathrm{C} 14$

$\mathrm{O} 1-\mathrm{N} 2-\mathrm{C} 3-\mathrm{C} 21$

$\mathrm{C} 7-\mathrm{N} 2-\mathrm{C} 3-\mathrm{N} 4$

$\mathrm{C} 7-\mathrm{N} 2-\mathrm{C} 3-\mathrm{C} 14$

$\mathrm{C} 7-\mathrm{N} 2-\mathrm{C} 3-\mathrm{C} 21$

$\mathrm{O} 1-\mathrm{N} 2-\mathrm{C} 7-\mathrm{C} 6$

$\mathrm{O} 1-\mathrm{N} 2-\mathrm{C} 7-\mathrm{C} 8$

$\mathrm{C} 3-\mathrm{N} 2-\mathrm{C} 7-\mathrm{C} 6$

$\mathrm{C} 3-\mathrm{N} 2-\mathrm{C} 7-\mathrm{C} 8$

$\mathrm{C} 5-\mathrm{N} 4-\mathrm{C} 3-\mathrm{N} 2$

$\mathrm{C} 5-\mathrm{N} 4-\mathrm{C} 3-\mathrm{C} 14$

$\mathrm{C} 5-\mathrm{N} 4-\mathrm{C} 3-\mathrm{C} 21$

C6-N4- $33-\mathrm{N} 2$

C6-N4- $33-\mathrm{C} 14$

C6-N4-C3-C21

$\mathrm{C} 3-\mathrm{N} 4-\mathrm{C} 6-\mathrm{O} 13$

$\mathrm{C} 3-\mathrm{N} 4-\mathrm{C} 6-\mathrm{C} 7$

C5-N4-C6-O13

$\mathrm{C} 5-\mathrm{N} 4-\mathrm{C} 6-\mathrm{C} 7$

$\mathrm{N} 2-\mathrm{C} 3-\mathrm{C} 14-\mathrm{C} 15$

$\mathrm{N} 2-\mathrm{C} 3-\mathrm{C} 14-\mathrm{C} 18$

$\mathrm{N} 4-\mathrm{C} 3-\mathrm{C} 14-\mathrm{C} 15$

N4-C3-C14-C18

$\mathrm{C} 21-\mathrm{C} 3-\mathrm{C} 14-\mathrm{C} 15$
$\mathrm{C} 14-\mathrm{C} 3-\mathrm{C} 21-\mathrm{C} 20$

$\mathrm{O} 13-\mathrm{C} 6-\mathrm{C} 7-\mathrm{N} 2$

$\mathrm{O} 13-\mathrm{C} 6-\mathrm{C} 7-\mathrm{C} 8$

N4-C6-C7-N2

$\mathrm{N} 4-\mathrm{C} 6-\mathrm{C} 7-\mathrm{C} 8$

$\mathrm{N} 2-\mathrm{C} 7-\mathrm{C} 8-\mathrm{C} 9$

$\mathrm{N} 2-\mathrm{C} 7-\mathrm{C} 8-\mathrm{C} 12$

$\mathrm{C} 6-\mathrm{C} 7-\mathrm{C} 8-\mathrm{C} 9$

$\mathrm{C} 6-\mathrm{C} 7-\mathrm{C} 8-\mathrm{C} 12$

$\mathrm{C} 7-\mathrm{C} 8-\mathrm{C} 9-\mathrm{O} 1$

$\mathrm{C} 7-\mathrm{C} 8-\mathrm{C} 9-\mathrm{C} 10$

$\mathrm{C} 12-\mathrm{C} 8-\mathrm{C} 9-\mathrm{O} 1$

$\mathrm{C} 12-\mathrm{C} 8-\mathrm{C} 9-\mathrm{C} 10$

$\mathrm{C} 7-\mathrm{C} 8-\mathrm{C} 12-\mathrm{O} 11$

C9-C8-C12-O11

$\mathrm{O} 1-\mathrm{C} 9-\mathrm{C} 10-\mathrm{O} 11$

$\mathrm{C} 8-\mathrm{C} 9-\mathrm{C} 10-\mathrm{O} 11$

$\mathrm{C} 3-\mathrm{C} 14-\mathrm{C} 15-\mathrm{C} 16$

$\mathrm{C} 3-\mathrm{C} 14-\mathrm{C} 15-\mathrm{C} 17$

$\mathrm{C} 18-\mathrm{C} 14-\mathrm{C} 15-\mathrm{C} 16$

$\mathrm{C} 18-\mathrm{C} 14-\mathrm{C} 15-\mathrm{C} 17$

C3-C14-C18-C19

$\mathrm{C} 15-\mathrm{C} 14-\mathrm{C} 18-\mathrm{C} 19$

$\mathrm{C} 14-\mathrm{C} 18-\mathrm{C} 19-\mathrm{C} 20$

$\mathrm{C} 18-\mathrm{C} 19-\mathrm{C} 20-\mathrm{C} 21$

$\mathrm{C} 18-\mathrm{C} 19-\mathrm{C} 20-\mathrm{C} 22$

$\mathrm{C} 19-\mathrm{C} 20-\mathrm{C} 21-\mathrm{C} 3$

$\mathrm{C} 22-\mathrm{C} 20-\mathrm{C} 21-\mathrm{C} 3$
$-57.7(2)$

$-169.1(2)$

$-51.0(3)$

$13.2(2)$

$131.3(2)$

$9.3(2)$

$-100.5(2)$

$-108.1(2)$

$142.1(2)$

$19.2(2)$

$-102.2(2)$

$137.68(18)$

$16.3(2)$

$74.2(2)$

$-35.1(2)$

$-107.8(2)$

$7.6(2)$

146.3 (2)

$-90.4(2)$

-84.9 (2)

38.4 (3)

$-54.8(3)$

$174.06(19)$

55.5 (3)

$-55.6(3)$

-177.5 (2)

58.7 (2)

-178.24 (19)

Hydrogen-bond geometry $\left(A,{ }^{\circ}\right)$

\begin{tabular}{lllll}
\hline$D-\mathrm{H} \cdots A$ & $D-\mathrm{H}$ & $\mathrm{H} \cdots A$ & $D \cdots A$ & $D-\mathrm{H} \cdots A$ \\
\hline $\mathrm{C} 5-\mathrm{H} 52 \cdots \mathrm{O} 13^{\mathrm{i}}$ & 0.97 & 2.57 & $3.536(3)$ & 172 \\
\hline
\end{tabular}

Symmetry code: (i) $-x+1, y-1 / 2,-z+1 / 2$. 